九州大学学術情報リポジトリ

Kyushu University Institutional Repository

\title{
On a Non-parametric Test in Life Test
}

Ishi i, Goro

Ikuno Senior High School

https://doi.org/10.5109/12985

出版情報 : 統計数理研究. 8 (3/4)，pp.73-79，1959-03. Research Association of Statistical Sciences

バージョン :

権利関係 : 


\title{
ON A NON-PARAMETRIC TEST IN LIFE TEST
}

\author{
By \\ Gorô ISHII \\ (Received November 30, 1958)
}

$\S$ 1. Introduction. There are various topics in the theory of life test. The purpose of this paper is to treat the problem from the standpoint of " test of fit." In life test we start with $M$ items and stop the test at a certain preassigned time $T$ or stop when the $s$-th death has occurred, because if we wait till all the death occur we have to wait for a long time, c.f. B. Epstein [2], [3], G. Ishii [5]. In a previous paper, we had treated the test of fit in life test in the case that we stop when the $s$-th death has occurred, G. Ishii [4.]. In the present paper we treat the case when we stop the life test at a certain preassigned time $T$.

Let $X$ be a random variable having the continuous distribution function. In order to test the hypothesis $H_{0}$ that the distribution function is a known function $F(x)$, F. N. David [1] and M. Okamoto [6] have proposed the following non-parametric test:

Let $x_{i}(i=1,2, \cdots, M)$ be $M$ independent observation of a random variable $X$. There are real numbers $\left\{a_{i}\right\} i=1,2, \cdots, m-1$ such that $F\left(a_{i}\right)$ $-F\left(a_{i-1}\right)=1 / m, i=1,2, \cdots, m$ where $a_{0}=-\infty, a_{m}=+\infty$. ( $\left.a_{i-1}, a_{i}\right]$ will be called "part." Let $v$ be the number of parts which contain no $x$ 's. If $v$ is too large, we reject $H_{0}$.

Now we shall apply the above non-parametric test to life test.

$\S 2$. Distribution of $v$ in life test. In the following, we shall treat the life test in the case that we stop the test at a certain preassigned time $T$, where $F(T)=t$. We call $T$ 'stop time.' We take the stop time $T$ as is equal to a divided point $a_{n}$ such that $F(T)=F\left(a_{n}\right)=t=n / m$. We start the life test with $M$ items and stop at time $T$. Suppose that there are $N$ deaths. Then $N$ is a random variable which follows the binomial distribution.

$$
P(\Re=N)=\left(\begin{array}{c}
M \\
N
\end{array}\right) t^{N}(1-t)^{M-N}
$$

$N$ observations are situated in some $\left(-\infty, a_{1}\right],\left(a_{1}, a_{2}\right], \cdots,\left(a_{n-1}, T\right]$. Let $v$ be the number of parts which contain no $x$ 's in the above $n$ parts.

For fixed $N$, we have

$$
P(v=\nu \mid \Re=N)=n^{-N}\left(\begin{array}{l}
n \\
\nu
\end{array}\right) \sum_{k=1}^{n-\nu}(-1)^{n-\nu-k}\left(\begin{array}{c}
n-\nu \\
k
\end{array}\right) k^{N} .
$$


Then we have

$$
P(v=\nu, \mathfrak{R}=N)=P(v=\nu \mid \mathfrak{R}=N) \cdot P(\mathfrak{N}=N) .
$$

We put $v^{(s)}=v(v-1) \cdots(v-s+1)$.

Then the $s$-th factorial moment is written as

$$
E\left(v^{(s)}\right)=\frac{n !}{(n-s) !}\left(1-\frac{s}{m}\right)^{M} .
$$

Putting $s=1,2$,

$$
\begin{aligned}
& E(v)=n(1-1 / m)^{M}, \\
& E(v(v-1))=n(n-1)(1-2 / m)^{M} .
\end{aligned}
$$

If $M \rightarrow \infty, m \rightarrow \infty, n \rightarrow \infty$ under the restriction of $M=m r$ ( $r$ is a constant) and $n=m t$ ( $t$ is a constant),

$$
\begin{aligned}
& E(v / n)=e^{-r}(1-r / 2 m)+O\left(m^{-2}\right), \\
& D^{2}(v / n)=e^{-2 r}\left(e^{r}-1-t r\right) / n+O\left(m^{-2}\right) .
\end{aligned}
$$

Under the above conditions we have next theorem.

Theorem 1. $v / n$ is asymptotically normally distributed with mean $e^{-r}$ and variance $e^{-2 r}\left(e^{r}-1-t r\right) / n$, where $M=m r, n=m t, \quad(r$ and $t$ are constants).

Proof. It is sufficient to prove that the moments of $(n / c)^{1 / 2}\left(v / n-e^{-r}\right)$ Lend to the moments of the standard normal distribution, where $c=e^{-2 r}\left(e^{r}\right.$ $-1-t r)$. The proof is almost parallel to that of theorem 1 of M. Okamoto [6], and theorem 2 of B. Sherman [7].

Denoting by $B_{r}^{(n)}$ the Bernoulli's number of order $\boldsymbol{n}$ and degree $\boldsymbol{r}$.

(9) $E\left\{\left(\frac{n}{c}\right)^{1 / 2}\left(\frac{v}{n}-e^{-r}\right)\right\}^{l}=\left(\frac{n}{c}\right)^{l / 2} \sum_{k=0}^{l}\left(\begin{array}{l}l \\ k\end{array}\right)\left(-e^{-r}\right)^{l-k} E\left(\frac{v}{n}\right)^{k}$

$$
\begin{aligned}
& =\left(\frac{n}{c}\right)^{l / 2} \sum_{k=0}^{l}\left(\begin{array}{l}
l \\
k
\end{array}\right)\left(-e^{-r}\right)^{l-k} n^{-k} \sum_{q=0}^{k}\left(\begin{array}{l}
k \\
q
\end{array}\right) B_{q}^{(q-k)} E\left(v^{(k-q)}\right) \\
& =\left(\frac{n}{c}\right)^{l / 2} \sum_{k=0}^{l} \sum_{q=0}^{k} \frac{l !(-1)^{l-k} e^{-r(l-k)}}{k !(l-k) !} \frac{k !}{q !(k-q) !} B_{q}^{(q-k)} \frac{n !(m-k+q)^{M}}{(n-k+q) ! m^{M} n^{k}} \\
& =\frac{(-1)^{l} n^{l / 2} l !}{\left(e^{r}-1-t r\right)^{l / 2}}\left\{a_{0}+\frac{a_{1}}{n}+\frac{a_{2}}{n^{2}}+\cdots+\frac{a_{\alpha}}{n^{\alpha}}+\cdots\right\} .
\end{aligned}
$$

We have to evaluate these $a_{i}$. In the above expansion

$$
\begin{aligned}
\frac{n !(m-k+q)^{M}}{(n-k+q) ! m^{M} n^{k}} & =\frac{1}{n^{k}}\left(1-\frac{k-q}{m}\right)^{M} n(n-1) \cdots(n-k+q+1) \\
& =n^{-q}\left(1-\frac{k-q}{m}\right)^{M}\left(1-\frac{1}{n}\right)\left(1-\frac{2}{n}\right) \cdots\left(1-\frac{k-q-1}{n}\right)
\end{aligned}
$$




$$
\begin{aligned}
& =x^{q}\{1-(k-q) t x\}^{M^{k-q-1}} \prod_{j=1}(1-j x) \\
& =x^{q} \cdot G(x)
\end{aligned}
$$

where

$$
\begin{aligned}
& x=1 / n \\
& G(x)=\{1-(k-q) t x)\}^{\frac{r}{x t}} \prod_{j=1}^{k-q-1}(1-j x) .
\end{aligned}
$$

Expanding this, we have

$$
G(x)=a_{k q 0}+a_{k q 1} x+a_{k q 2} x^{2}+\cdots,
$$

where

(11)

$$
\begin{aligned}
a_{k q 0}= & e^{-(k-q) r} \\
a_{q q p}= & \frac{1}{p !}\left\{\frac{d^{p} G(x)}{d x^{p}}\right\}_{x=0} \\
= & \left.\frac{1}{p !} \sum_{s=0}^{p-1}\left(\begin{array}{c}
p-1 \\
s
\end{array}\right) \frac{d^{p-1} G(x)}{d x^{p-1}} \frac{d^{s+1} \log G(x)}{d x^{s+1}}\right|_{x=0} \\
\log G(x)= & -\sum_{j=1}^{k-q-1}\left(j x+\frac{j^{2} x^{2}}{2}+\frac{j^{3} x^{3}}{3}+\cdots+\frac{j^{s+1} x^{s+1}}{s+1}+\cdots\right) \\
& \quad-\frac{r}{x t}\left((k-q) t x+\frac{(k-q)^{2} t^{2} x^{2}}{2}+\cdots+\frac{(k-q)^{s+2} t^{s+2} x^{s+2}}{s+2}+\cdots\right) .
\end{aligned}
$$

Therefore

$$
\begin{aligned}
\left.\frac{d^{s+1} \log G(x)}{d x^{s+1}}\right|_{x=0} & =-\sum_{j=1}^{k-q-1} s ! j^{s+1}-\frac{(s+1) !(k-q)^{s+2}}{s+2} r t^{s+1} \\
& =s ! b_{k q s} \quad(\mathrm{say}) \\
b_{k q s} & =-\sum j^{s+1}-\frac{s+1}{s+2} r t^{s+1}(k-q)^{s+2}
\end{aligned}
$$

Then $b_{k q s}$ is a polynomial in $k$ of degree $s+2$.

Putting $s=0$,

$$
\begin{aligned}
b_{k q 0} & =-\frac{(k-q)(k-q-1)}{2}-\frac{1}{2} r t(k-q)^{2} \\
& =-(1+r t) \frac{k^{2}}{2}+A k+B \quad \text { (say). }
\end{aligned}
$$

From (11),

$$
\begin{aligned}
a_{k p q} & =\frac{1}{p !} \sum_{s=0}^{p-1} \frac{(p-1) !}{s !(p-s-1) !} a_{k q(p-1-s)}(p-s-1) ! s ! b_{k q s} \\
& =\frac{1}{p} \sum_{s=0}^{p-1} a_{k q(p-1-s)} b_{k q s} .
\end{aligned}
$$


Then we have next equations

$$
\begin{aligned}
& a_{k q 0}=e^{-(k-q) s} \\
& a_{k q 0} b_{k q 0}-a_{k q 1}=O \\
& a_{k q 0} b_{k q 1}+a_{k q 1} b_{k q 0}-2 a_{k q 2}=O \\
& \ldots \ldots \ldots \ldots \ldots \ldots \ldots \ldots \ldots \ldots \ldots \ldots \ldots \ldots \ldots \\
& a_{k q 0} b_{k q(i-q-1)}+a_{k q 1} b_{k q(i-q-2)}+\cdots+a_{k q(i-q-1)} b_{k q 0}-(i-q) a_{k q(i-q)}=O .
\end{aligned}
$$

Hence we have

$$
a_{k q(i-q)}=e^{-(k-q) r} B_{k q i},
$$

where

$$
\begin{aligned}
B_{k q i} & =\frac{\left(b_{k q 0}\right)^{i-q}}{(i-q) !}+(\text { terms of lower degree in } k) \\
& =\frac{1}{(i-q) !}\left(-\frac{1+r t}{2}\right)^{i-q} k^{2(i-q)}+\sum_{j=0}^{2(i-q)-1} A_{j} k^{j}
\end{aligned}
$$

In (9) we put

$$
\begin{aligned}
& \sum_{q=0}^{l} \sum_{k=q}^{l} \frac{(-1)^{k} e^{r k}}{q !(l-k) !(k-q) !} B_{q}^{(q-k)} \frac{n !(m-k+q)^{M}}{(n-k+q) ! m^{M} n^{k}} \\
= & a_{0}+\frac{a_{1}}{n}+\frac{a_{2}}{n^{2}}+\cdots+\frac{a_{\alpha}}{n^{\alpha}}+\cdots .
\end{aligned}
$$

If we denote by $a_{i q}$ the coefficient of $n^{-i}$ in the expansion in the power of $n^{-1}$ of

$$
\begin{aligned}
& \sum_{k=q}^{i} \frac{(-1)^{k} e^{r k}}{(l-k) !(k-q) !} B_{q}^{(q-k)} \frac{n !(m-k+q)^{M}}{(n-k+q) ! m^{M} n^{k}} \\
= & \sum_{k=q}^{l} \frac{(-1)^{k} e^{r k}}{(l-k) !(k-q) !} B_{q}^{(q-k)} x^{q}\left\{a_{k q 0}+a_{k q 1} x+\cdots+a_{k q(i-q)} x^{i-q}+\cdots\right\},
\end{aligned}
$$

we have

$$
a_{i}=\sum_{q=0}^{l} \frac{a_{i q}}{q !}
$$

and

$$
\begin{aligned}
a_{i q} & =\sum_{k=q}^{l} \frac{(-1)^{k} e^{r k}}{(l-k) !(k-q) !} B_{q}^{(q-k)} a_{i q(i-q)} \\
& =e^{q r} \sum_{k=q}^{l} \frac{(-1)^{k}}{(l-k) !(k-q) !} B_{q}^{(k-b)} B_{k q i} .
\end{aligned}
$$

$B_{q}^{(q-k)}$ is the coefficient of the expansion of $\left(q /\left(e^{t}-1\right)\right)^{q-k}$, then $B_{q}^{(q-k)}$ is the polynomial in $k$ of degree $q$ with $2^{-q}$ as the coefficient of the term of highest degree. Therefore 
(17)

$$
\begin{aligned}
& a_{i q}=e^{q r} \sum_{k=q}^{l} \frac{(-1)^{k}}{(l-k) !(k-q) !}\left\{\frac{k^{q}}{2^{q}}+\cdots\right\}\left\{\frac{k^{2(i-q)}}{(i-q) !}\left(-\frac{1+t r}{2}\right)^{i-q}+\sum A_{j} k^{j}\right\} \\
= & \frac{e^{q r}}{2^{q}(i-q) !}\left(-\frac{1+t r}{2}\right)^{i-q} \sum_{k=q}^{l} \frac{(-1)^{k}}{(l-k) !(k-q) !}\left\{k^{2 i-q}+\begin{array}{l}
\text { terms of lower } \\
\text { degree in } k .
\end{array}\right\} .
\end{aligned}
$$

As

$$
\sum_{k=q}^{\prime} \frac{(-1)^{l} k^{l^{\prime}}}{(l-k) !(k-q) !}= \begin{cases}0 & l^{\prime}<l-q \\ 1 & l^{\prime}=l-q\end{cases}
$$

we obtain

$$
\begin{aligned}
& a_{i q}=0 \quad i<\frac{l}{2} \\
& a_{h q}=\frac{e^{q r}\left(-\frac{1+r t}{2}\right)^{i-q}}{2^{q}(h-q) !} \quad i=\frac{l}{2}=h \quad \text { (when } l \text { is even). }
\end{aligned}
$$

Thus from (16)

$$
\begin{aligned}
a_{i} & =0 \quad \text { when } \quad i<\frac{l}{2} \\
a_{h} & =\sum_{q=0}^{2 h} \frac{a_{h q}}{q !}=\sum_{q=0}^{2 h} \frac{1}{q !(h-q) !}\left(\frac{e^{r}}{2}\right)^{q}\left(-\frac{1+t r}{2}\right)^{h-q} \\
& =\frac{1}{h !} \frac{\left(e^{r}-1-\dot{t} r\right)^{h}}{2^{h}} .
\end{aligned}
$$

Then we have

$$
\begin{aligned}
& \lim _{n \rightarrow \infty} E\left\{\left(\frac{n}{c}\right)^{1 / 2}\left(\frac{v}{n}-e^{-r}\right)\right\}^{l}=0 \quad \text { (when } l \text { is odd) } \\
& \lim _{n \rightarrow \infty} E\left\{\left(\frac{n}{c}\right)^{1 / 2}\left(\frac{v}{n}-e^{-r}\right)\right\}^{2 n}=\frac{a_{h}(2 h) !}{\left(e^{r}-1-t r\right)^{n}}
\end{aligned}
$$

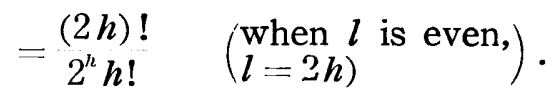

This completes the proof.

$\S 3$. The power function. The power function of the test with respect to the alternative hypothesis $H_{1}$ is

$$
P=P\left(v \geq l\left(H_{1}\right)\right. \text {. }
$$

Under $H_{1}$ : distribution function is $F_{1}(x)$, and the range of distribution is equal to that of $F(x)$, where $F(T)=\tau$

$$
\begin{aligned}
& \qquad \int_{i-1 / m}^{i / m} d H(x)=p_{i}, \quad i=1,2, \cdots, n \\
& \text { where } \quad H(x)=F_{1}\left(F^{-1}(x)\right), \quad \sum_{i=1}^{n} p_{i}=\tau .
\end{aligned}
$$


We put $p_{i} / \tau=q_{i}$, then $\sum_{i=1}^{n} q_{i}=1$

$$
\begin{aligned}
& P\left(\Re=N \mid H_{1}\right)=\left(\begin{array}{c}
M \\
N
\end{array}\right) \tau^{N}(1-\tau)^{M-N} \\
& P\left(v=k|\mathfrak{N}=N| H_{1}\right)=\sum_{j=1}^{n-k}(-1)^{n-k-j}\left(\begin{array}{c}
n-j \\
k
\end{array}\right) \sum_{\left(i_{1} \cdots i_{j}\right)}^{n}\left(q_{i_{1}}+\cdots+q_{i_{j}}\right)^{N},
\end{aligned}
$$

where $\sum_{\left(t_{1} \cdots t_{l}\right)}^{i}$ denotes the summation over all combinations $\left(t_{1}, \cdots, t_{l}\right)$ drawn from $(1,2, \cdots, j)$.

$$
\begin{aligned}
P(v=k, \mathfrak{N} & \left.=N \mid H_{1}\right)=P\left(v=k|\mathfrak{N}=N| H_{1}\right) \cdot P\left(\mathfrak{N}=N \mid H_{1}\right) \\
E\left(v^{(s)} \mid H_{1}\right) & =\sum_{N} s ! \sum_{\left(i_{1} \cdots i_{s}\right)}^{n}\left(1-q_{i_{1}}-\cdots-q_{i_{s}}\right)^{N}\left(\begin{array}{c}
M \\
N
\end{array}\right) \tau^{N}(1-\tau)^{M-N} \\
& =s ! \sum_{\left(i_{1} \cdots i_{s}\right)}^{n}\left(1-p_{i_{1}}-\cdots-p_{i_{s}}\right)^{\prime \prime} \cdot
\end{aligned}
$$

Putting $s=1$, 2, we get

$$
\begin{aligned}
& E\left(v \mid H_{1}\right)=\sum_{i=1}^{n}\left(1-p_{i}\right)^{M} \\
& E\left(v(v-1) \mid H_{1}\right)=\sum_{i \neq j}^{n}\left(1-p_{i}-p_{j}\right)^{M} .
\end{aligned}
$$

If $F_{1}(x)$ is absolutely continuous with respect to $F(x)$ and its relative density is differentiable, putting $H^{\prime}(x)=h(x)$,

$$
\begin{aligned}
& p_{i}=\frac{1}{m} h\left(\frac{i}{m}\right)-\frac{1}{2 m^{2}} h^{\prime}\left(\frac{i}{m}\right)+O\left(m^{-3}\right) \\
& \left(1-p_{i}\right)^{M}=e^{-r h\left(\frac{i}{m}\right)}\left[1+\frac{r}{2 m}\left\{h^{\prime}\left(\frac{i}{m}\right)-h^{2}\left(\frac{i}{m}\right)\right\}+O\left(m^{-2}\right)\right] \\
& E\left(v / n \mid H_{1}\right)=\frac{1}{t} \int_{0}^{t} e^{-r h(x)} d x-\frac{r}{2 m t} \int_{0}^{t} e^{-r h(x)} h^{2}(x) d x+O\left(m^{-2}\right) .
\end{aligned}
$$

The power function

$$
P=\sum_{k \geqq l} \sum_{N=0}^{M} P\left(v=k, \mathfrak{R}=N \mid H_{1}\right)
$$

satisfies the next condition when $\tau \leqq t$

$$
P \geqq P\left(v \geqq l \mid H_{0}\right) \text {. }
$$

This inequality can be proved by the same method in theorem 3 of Oka. moto [6].

Thus we have the next theorem.

Theorem 2. The test based on $v$ is unbiased against the class of alternative hypotheses, $\tau \leqq t$. 
Acknowledgement. The author is deeply indebted to Mr. M. Okamoto whose helpful guidance made this work possible.

\section{IKUNO SENIOR HIGH ScHOOL*}

\section{References}

[1] F. N. DAVID; "Two combinatorial tests of whether a sample has come from a given population" Biometrika, vol. 37 (1950), pp. 97-110.

[2] B. Epstein; "Truncated life tests in the exponential case" Ann. Math. Stat., vol. 25 (1954), pp. 555-64.

[3] B. EPSTEIN ; "Simple estimator of the parameter of exponential distributions when sample are censored" Ann. Inst. Stat. Math., vol. 8 (1956-7), pp. 15-26.

[4] G. Isнi1 ; "Test of fit in life test" Ann. Inst. Stat. Math., vol. 9 (1957-8) pp. 117-125.

[5] G. Ishiг; "Life test in two dimensional exponential distribution" in Japanese. Tokei Kagaku Kenkyu, vol. 2 (1958).

[6] М. Окамото; "On a non-parametric test" Osaka Math. J., vol. 4 (1952), pp. 77-85.

[7] B. Sherman; "A random variable related to the spacing of sample values" Ann. Math. Stat., vol. 22 (1950), pp. 339-361.

[8] G. IsHi ; “Kolmogorov-Smirnov test in life test” Ann. Inst. Stat. Math., vol. 10 (1958-9) pp. 3746.

[9] I. WeIss; "Limiting distribution in some cccupancy problem" Ann. Math. Stat., vol. 29 (1958) pp. $878-884$.

* Present address: Atomic Bomb Casualty Commission, Hiroshima. 